\title{
Um Estudo Numérico da Intensificação do Vento em Reservatórios de Centrais Hidroelétricas da Região Sudeste do Brasil
}

A numerical study of Wind speed-up over hydroelectric reservoirs of the Southeast Region of Brazil

\author{
Claudio C. Pellegrini ${ }^{1}$, Almilson V. L. Neto ${ }^{2}$, João Victor B. Assis ${ }^{2}$, Arcilan T. Assireu ${ }^{3}$, \\ ${ }^{1}$ D.Sc., DCTEF, Univ. Fed. de São João del-Rei (UFSJ), São João del-Rei, MG, Brasil \\ ccpellegrini@gmail.com \\ ${ }^{2}$ Mestrando, PPGEE, Univ. Fed. de São João del-Rei (UFSJ), São João del-Rei, MG, Brasil \\ almilson@hotmail.com; joaovictorassis@hotmail.com \\ ${ }^{3}$ D.Sc., IRN, Univ. Fed. de Itajubá,Itajubá, MG, Brasil \\ assireu@gmail.com
}

\begin{abstract}
Resumo
Em solo brasileiro há um enorme potencial hídrico explorado de diversas formas, destacando-se a geração hidrelétrica. Entretanto, estudos sobre intensificação e convergência do vento, influenciados pelo relevo e baixa rugosidade da superfície dos reservatórios, ainda são escassos. Propôs-se neste trabalho uma avaliação do fenômeno, mediante dados observacionais e simulações numéricas com o modelo atmosférico WRF. Os reservatórios selecionados para averiguação estão localizados na região SE e possuem infra-estrutura de transmissão operante. Com vistas neste fato, procurou-se entender a influência do conjunto reservatório-relevo sobre o comportamento do vento. No reservatório de Itumbiara (GO) foi verificado que o vento sofre intensificação tanto em regime de alinhamento quanto de incidência transversal aos braços do reservatório. Já no reservatório de Três Marias (MG), a análise mostrou que o vento também é intensificado quando alinhado, porém em alguns casos de não alinhamento, a intensificação não é significativa. Concluiu-se que para ambos os reservatórios há aceleração do vento causada pela ação do conjunto reservatório-relevo, mais pronunciada a partir de 3,0 m/s (10 m do solo), velocidade considerada de entrada (cut-in) para as turbinas eólicas modernas.
\end{abstract}

Palavras-chave: Potencial eólico, Modelagem de mesoescala, WRF, reservatórios hidroelétricos.

\begin{abstract}
Brazil has a large hydric potential explored in many ways, especially for electrical generation. However there is a lack of studies about the wind acceleration a converge over the reservoir, affected by the surrounding topography and the low roughness of the water surface. In this work an evaluation of the phenomenon is performed thought observed data and numerical simulation using the WRF atmospheric model. The selected reservoirs are located in the Brazilian south-east region, and both have operating infrastructure of transmission. In view of that, we have tried to understand the influence of the joint reservoir-topography over the wind behavior. It was verified that in the Itumbiara reservoir (Goias State), the wind experiences acceleration with both cross and aligned wind with respect to the reservoir branches. Otherwise, the Tres Marias reservoir (State of Minas Gerais) shows that in the case of non-aligned wind the reservoir has not a significant influence over wind speed. It is concluded that for both reservoirs wind speed-ups are verified, caused by the joint reservoir-topography influence, that becomes more evident with speeds over $3.0 \mathrm{~m} / \mathrm{s}$ (10 m over the ground) a velocity matching modern wind turbines cut out speed.
\end{abstract}

Keywords: Wind potential, Mesoscale modeling, WRF model, hydroelectric reservoirs. 


\section{Introdução}

O Brasil é um país de dimensões continentais e possui um enorme potencial hídrico distribuído nas diversas bacias hidrográficas espalhadas pelas regiões do país. Esse potencial é largamente explorado em localidades cujo relevo permite o represamento de afluentes volumosos para diversos fins como, por exemplo, abastecimento da população e/ou geração hidrelétrica.

Alguns reservatórios possuem grandes dimensões e criam uma extensa lamina d'água que, em conjunto com o relevo, proporciona zonas de intensificação e convergência do vento.

Em estudo observacional Assireu et al. (2011) coletaram dados da intensidade e persistência do vento em grandes reservatórios localizados em diversas regiões do país. Foi verificado que em todos há convergência e intensificação dos ventos alinhados com os braços principais dos reservatórios. No reservatório de Itumbiara (GO), por exemplo, foi verificado que em $93 \%$ do tempo o vento registrado a $100 \mathrm{~m}$ era superior a $3 \mathrm{~m} / \mathrm{s}$ e que a média era de $7,9 \mathrm{~m} / \mathrm{s}$. Além disso, os ventos de leste eram amplamente predominantes, atuando em mais que 35\% do tempo.

Assireu et al. (2013) realizaram um novo estudo da intensificação do vento especificamente na represa de Itumbiara (GO) comparando dados observados com resultados numéricos do modelo WRF e concluíram que, mesmo ventos não alinhados com a direção principal do reservatório sofrem uma intensificação devido a influência complexa do relevo e da lâmina d'água.Este efeito da intensificação dos ventos em regiões de morfometria do tipo " $K$ " é bem conhecido e maiores detalhes podem ser encontrados em Bullard et al. (2000).

Assireu et al. (2011) avaliaram a característica dos ventos em oito reservatórios de geração hidrelétrica no intuito de verificar o possível potencial eólico para geração de eletricidade. Estudos dessa natureza são de extrema importância e devem ser bem explorados, por permitir que novas fontes energéticas sejam estabelecidas onde já existe infra-estrutura de transmissão instalada e operante.
O objetivo deste trabalho divide-se em duas partes: primeiramente avaliar a influência do relevo e do reservatório de Itumbiara (GO) sobre a convergência e intensificação dos ventos, comparando dados observados com resultados de simulação numérica do modelo WRF. Especificamente, obter meteogramas que mostrem a acurácia do modelo mediante comparação com os dados observados em períodos marcados pela alta e baixa intensidade do vento na região.

$\mathrm{Na}$ segunda parte, propõe-se realizar uma analise puramente numérica no reservatório de Três Marias (MG), para o qual não se dispõe no momento de dados observados, e verificar a influência do relevo e dos braços do mesmo sobre os ventos predominantes na região. O reservatório de Três Marias possui como característica braços tanto alinhados quanto perpendiculares ao vento de larga escala (predominantes do quadrante E segundo as Normas Climatológicas do Brasil, 1961-1990, INMET) e espera-se que as simulações lancem luz sobre a influência desta morfologia complexa sobre o comportamento do vento.

\section{Metodologia}

Dois reservatórios foram escolhidos para este estudo: Itumbiara (GO - anteriormente avaliado por Assireu et al., 2011) e Três Marias (MG). Para o primeiro, foram comparados dados anemométricos observados com os resultados de simulações numéricas no intuito de averiguar a capacidade do modelo em descrever o escoamento próximo à superfície do relevo complexo que caracteriza a região. Para o segundo, será analisada a relação do alinhamento e intensificação dos ventos com os braços do reservatório utilizando apenas resultados de simulações numéricas, uma vez que dados observacionais ainda não se encontram disponíveis no âmbito do projeto.

Em Itumbiara, dados da intensidade do vento foram obtidos mediante a utilização de uma boia SIMA (Sistema Integrado de Monitoramento Ambiental) instalada no reservatório, nas coordenadas $18^{\circ} 21^{\prime} 43^{\prime \prime} \mathrm{S}$ e $48^{\circ} 59^{\prime} 29^{\prime \prime} \mathrm{O}$. Os dados foram coletados a $3 \mathrm{~m}$ acima da lâmina d'água. 
Todas as simulações foram realizadas com a versão 3.5 do modelo WRF e, para ambos os reservatórios, foram escolhidos dois períodos: estação de ventos mais intensos (agosto) e de ventos menos intensos (abril). Estes períodos foram determinados com base em uma análise das Normas Climatológicas do Brasil, 1961-1990 (INMET) para a região do reservatório de Itumbiara. Dentro de cada período foram simulados intervalos de uma semana, conforme Tabela 1. Em ambos os casos, as primeiras seis horas foram descartadas da análise como tempo de acomodação do modelo.

Tabela 1 - Intervalos semanais estudados.

\begin{tabular}{c|c|c}
\hline Mês & Data de Início & Data de Término \\
\hline Abril & $18: 00$ 05-04-2009 & 00:00 13-04-2009 \\
\hline Agosto & $18: 00$ 21-08-2009 & $00: 00$ 29-08-2009 \\
\hline
\end{tabular}

A escolha do ano 2009 é justificada devido à disponibilização dos dados de vento coletados pela boia SIMA. Quanto às semanas, foram selecionadas aquelas com registros observacionais mais completos.

Escolheu-se uma configuração de domínios aninhados e centralizados, conforme ilustrado na Fig. 1.

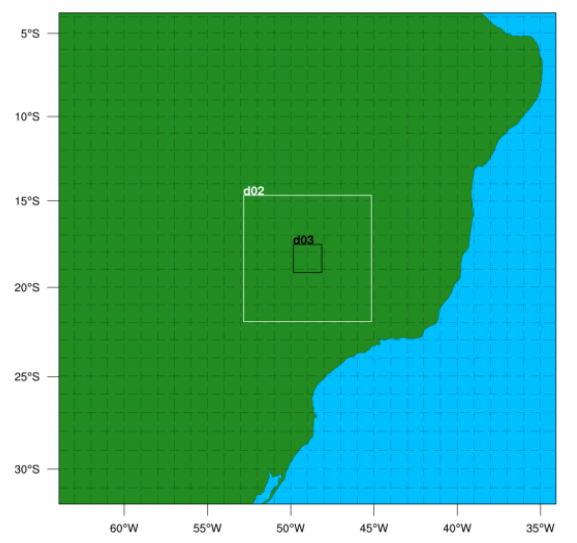

Figura 1 - Aninhamento dos domínios simulados em Itumbiara.

Para o reservatório de Itumbiara, a centralização da grade ocorreu em $18^{\circ} 21^{\prime} 43^{\prime \prime}$ S e $48^{\circ} 59^{\prime} 29^{\prime \prime}$ $\mathrm{O}$, coincidindo com a posição da boia. Em Três Marias, a centralização ocorreu em $18^{\circ} 38^{\prime} 12^{\prime \prime}$, $45^{\circ} 13^{\prime} 42^{\prime \prime} \mathrm{O}$, de forma a manter o reservatório no centro do domínio menor (Fig. 2). As características dos domínios para ambas as localidades encontram-se listadas na Tabela 2.

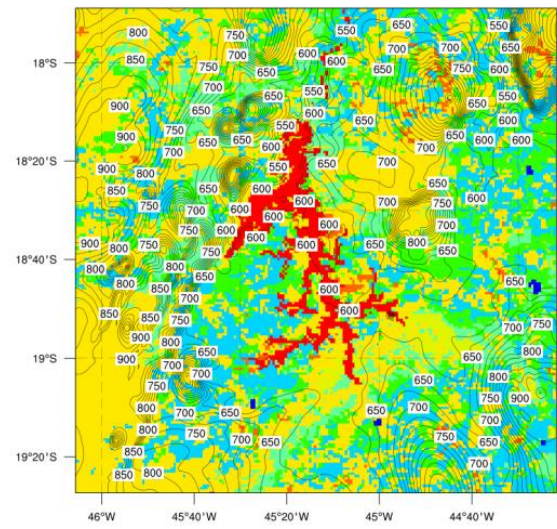

Figura 2 - Posicionamento do reservatório de Três Marias (em vermelho) no domínio menor.

Tabela 2 - Características dos domínios.

\begin{tabular}{c|c|c|c}
\hline Domínio & $\begin{array}{c}\text { Res. horizontal } \\
\mathbf{( k m )}\end{array}$ & $\begin{array}{c}\text { Pontos de } \\
\text { Grade }\end{array}$ & $\begin{array}{c}\text { Passo de } \\
\text { tempo (s) }\end{array}$ \\
\hline Dados FNL & 108 & - & 21.600 \\
\hline $\mathrm{d} 01$ & 22.5 & $141 \times 141$ & 120 \\
\hline $\mathrm{d} 02$ & 4.5 & $181 \times 181$ & 40 \\
\hline $\mathrm{d} 03$ & 0.9 & $201 \times 201$ & 13,333 \\
\hline
\end{tabular}

A resolução da grade menor, em ambos os casos, foi escolhida como $0,9 \mathrm{~km}$ de forma a coincidir com a resolução máxima disponível para os dados geográficos estáticos. A Fig. 2 ilustra a escolha para a variável uso do solo. Para aumentar progressivamente a resolução das grades aninhadas até a dos dados de inicialização, foi usada uma relação de 5:1 entre domínios. Com isso, chegou à configuração final de três domínios. Uma alta resolução vertical de 45 níveis com concentração maior próximo à superfície foi utilizada nos três domínios.

As parametrizações físicas usadas foram as mesmas em todos os domínios e estão listadas na Tabela 3. Os esquemas adotados foram os mais usualmente encontrados na literatura. A aplicabilidade destes modelos encontra-se bem documentada no manual do WRF

Todas as simulações foram inicializadas com os dados FNL operacionais globais de análise do modelo global GFS do NCEP (resolução de $1^{\circ}$ e 6 horas) também utilizados como condição de contorno ao longo da integração.

Para Itumbiara, uma correção da velocidade do vento observada para a altura de $10 \mathrm{~m}$ em relação à lâmina d'água foi feita, pois o WRF fornece dados de saída na altura padrão de $10 \mathrm{~m}$. Para tal correção foi utilizada a Lei de Potência conforme Manwell (2002). 


$$
V(z)=V_{a}\left(\frac{z}{z_{a}}\right)^{n}
$$

onde $V(z)$ é a velocidade no nível de interesse, $z$ o nível de interesse, $V_{a}$ a velocidade coletada e $z a$ a altura de coleta da velocidade. O expoente $n$, dependente das variáveis citadas acima, é dado por:

$$
n=\frac{\left[0,737-0,088 \ln V_{a}\right]}{\left[1-0,088 \ln \left(z_{a} / z\right)\right]}
$$

Tabela 3 - Parametrizações físicas utilizadas nas simulações em todos os domínios.

\begin{tabular}{c|c}
\hline Parametrização & Modelo \\
\hline Microfísica de Nuvens & Lin et al. Scheme \\
\hline Radiação de Onda Longa & Esquema RRTM \\
\hline Radiação de Onda Curta & Dudhia Scheme \\
\hline Camada Superficial & Eta Similarity Monin-Obukov \\
\hline Trocas com a superfície & Mellor-Yamada Scheme \\
\hline Nuvens Cúmulos & $\begin{array}{c}\text { Betts-Miller-Janjic Scheme em } \\
\text { d01 e d02; nenhuma em d03 }\end{array}$ \\
\hline
\end{tabular}

Por se tratar a Lei de Potência de um modelo que não leva em conta os efeitos da estabilidade atmosférica, o modelo acima não pode, a rigor, ser aplicado para estimativas do perfil vertical do vento em regiões de transição terra-água, como regiões costeiras ou offshore (Lange et al., 2004). Nestas regiões é necessária a utilização do modelo baseado na teoria da similaridade de Monin-Obukhov (SMO), que adiciona um termo de correção ao modelo clássico do perfil logarítmico (Peña et al., 2008; Peña et al., 2009; Sathe et al., 2011). Se este fato tem implicações importantes para a nossa região de estudo, está sob investigação (será comunicado em breve), porém fora do escopo do presente trabalho.

\section{Resultados e discussão}

A Figura 3 mostra o meteograma de velocidades a $10 \mathrm{~m}$ para Itumbiara no período de poucos ventos. No período inicial o modelo não descreve bem a intensidade observada do vento, mas a partir de 24 horas nota-se uma melhora. Dentro do período simulado alternam-se bons e maus períodos de previsão, mostrando que muito ainda existe para ser feito no sentido de melhorar a previsão dos ventos próximos à superfície em regiões de relevo complexo. Os resultados a seguir confirmam essa impressão.

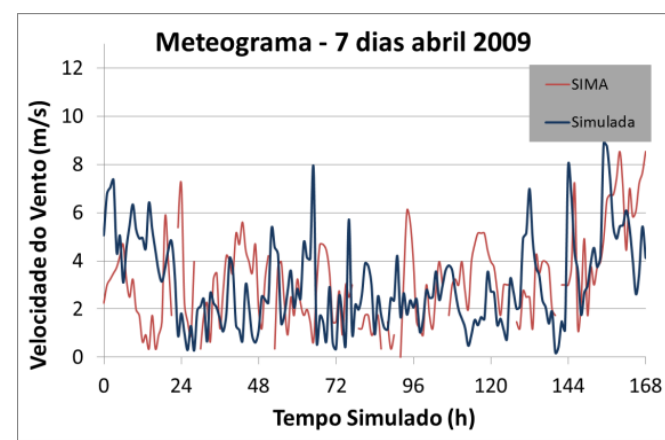

Figura 3 - Velocidade do vento a $10 \mathrm{~m}$, Itumbiara, mês de Abril.

A distribuição espacial média semanal da intensidade dos ventos (Fig. 4) mostra que a direção predominante no período simulado é NE e que não há alinhamento com o eixo principal do reservatório. Ainda assim, a aceleração é evidente, sugerindo que os ventos são canalizados pelo relevo ao redor. A velocidade média encontrada é aproximadamente $3,0 \mathrm{~m} / \mathrm{s}$, valor observado como mínimo em todo período de observação relatado por Assireu et al. (2011).

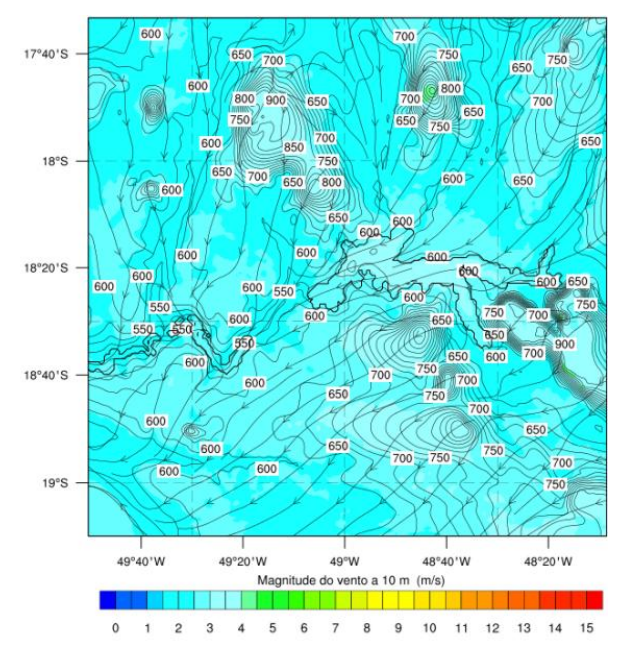

Figura 4 - Distribuição de velocidade média semanal a $10 \mathrm{~m}$, Itumbiara, abril. A linha cheia grossa mostra o contorno do reservatório.

Para o mês de ventos mais intensos (Fig. 5), nota-se a mesma tendência da Fig. 3.Neste período, os ventos predominantes de L estão alinhados com o eixo principal do reservatório (Fig. 6). A velocidade média encontrada é aproximadamente $7,0 \mathrm{~m} / \mathrm{s}$ e em alguns pontos atinge 10,0 $\mathrm{m} / \mathrm{s}$. É evidente o efeito de intensificação causado pelo relevo envolvente e pela lâmina d'água do reservatório, efeito já relatado por Assireu et al. (2013). 
Em ambos os períodos simulados, o braço alinhado com a direção sudeste, demarcado na Fig. 6, forma uma zona de desaceleração, possivelmente devido à recirculação do vento no local. O efeito mais fica claro em dias com vento de L e NE (não mostrados).

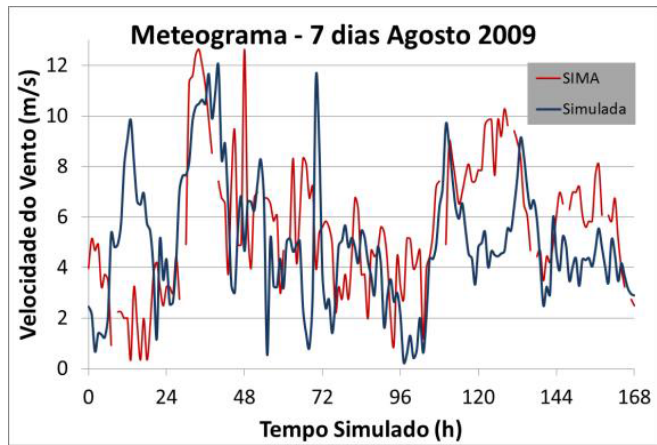

Figura 5 - Velocidade do vento a $10 \mathrm{~m}$, Itumbiara, mês de Agosto.

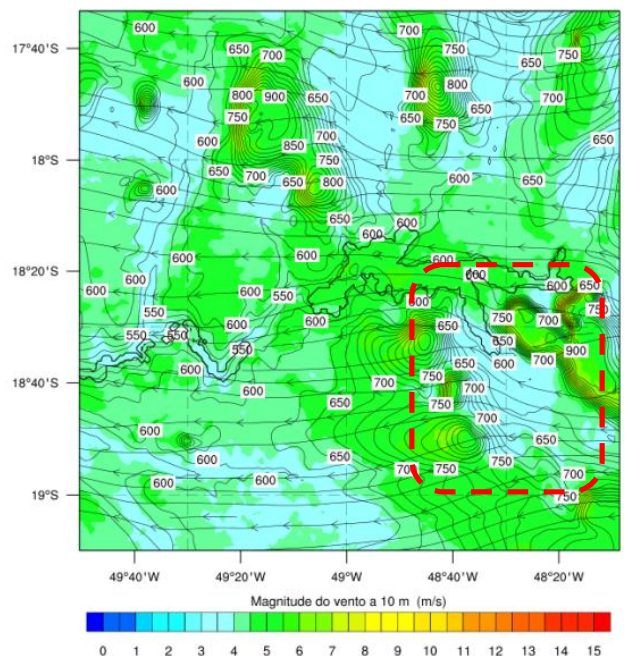

Figura 6 - Distribuição de velocidade média semanal a 10 m, Itumbiara, agosto.

Os resultados das simulações para o reservatório de Três Marias no mês de abril mostram que, na média semanal, não há uma direção predominante do vento no domínio simulado. Mesmo assim, sempre há aceleração do vento (Fig.7). Em agosto, a direção predominante do vento é SE e também não há um alinhamento pleno com o reservatório, porém, também é clara a intensificação do vento (Fig. 8).

Algumas peculiaridades foram notadas em dias específicos da simulação. O lago por vezes intensifica os ventos apenas tanto quanto o relevo ao seu redor. Notou-se também que durante a ocorrência de ventos $\mathrm{N}$, alinhados com o eixo principal do reservatório, o espelho d'água acelera bem mais o escoamento a $10 \mathrm{~m}$ (Fig. 9) do que em altitudes maiores (Fig.10, $80 \mathrm{~m}$ ). Por outro lado, em situações de vento S, (Fig. 11), mesmo este estando alinhado com o eixo principal do reservatório, não há aceleração perceptível, provavelmente devido à ausência do efeito canalizador do relevo.

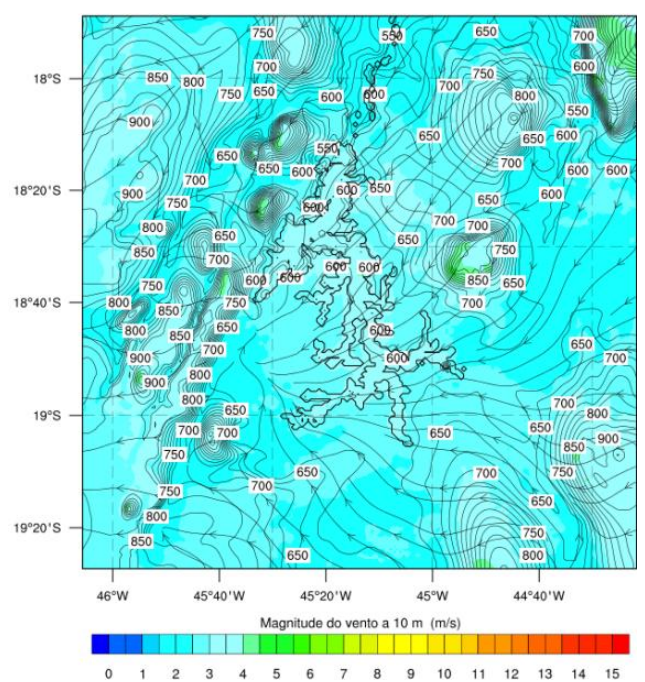

Figura 7- Distribuição de velocidade média semanal a 10 m, Três Marias, Abril.

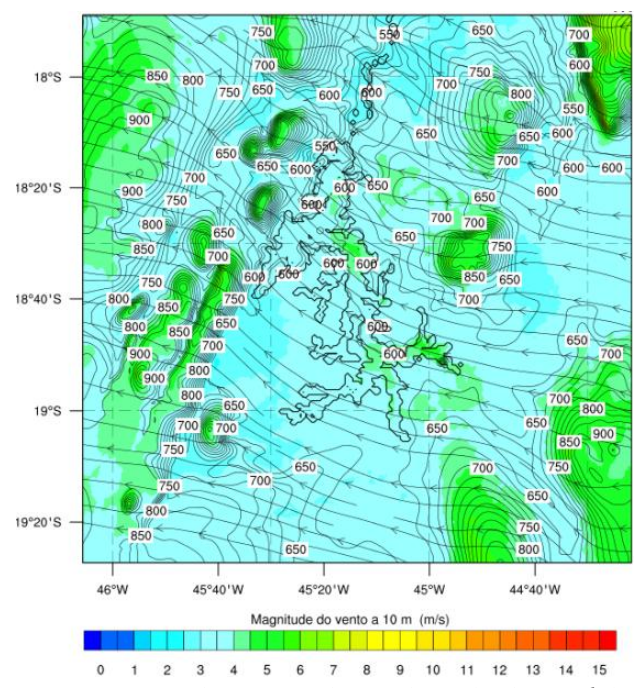

Figura 8- Distribuição de velocidade média semanal a 10 m, Três Marias, Agosto.

Percebe-se também que o relevo exerce forte influência sobre o comportamento dos ventos, por vezes anulando o efeito de intensificação resultante na pouca rugosidade superficial do lago. Este efeito se evidencia, por exemplo, ao analisar a distribuição do vento no dia 3 (Fig.12). Percebe-se que a parte central da represa tem braços alinhados com o vento (NE neste dia), mas não se nota uma intensificação do mesmo, enquanto em um braço ao $S$ o efeito se verifica. Pode-se supor que o responsável pela intensifi- 
cação sejam as colinas existentes na porção sudeste do domínio, que canalizam o vento. Por outro lado, provavelmente, a presença de uma elevação do relevo a NE do centro do reservatório age como um obstáculo à passagem do vento cancelando o efeito acelerador deste.

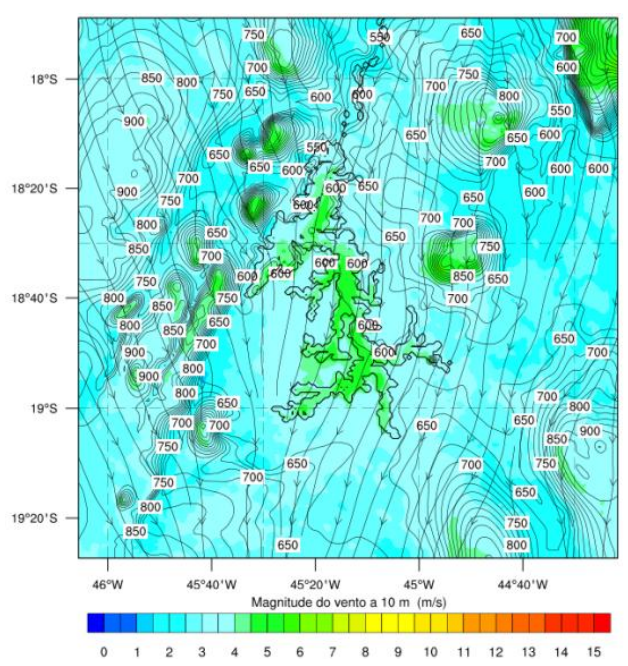

Figura 9 - Distribuição de velocidades, dia 2,10 m, Três Marias, Abril.

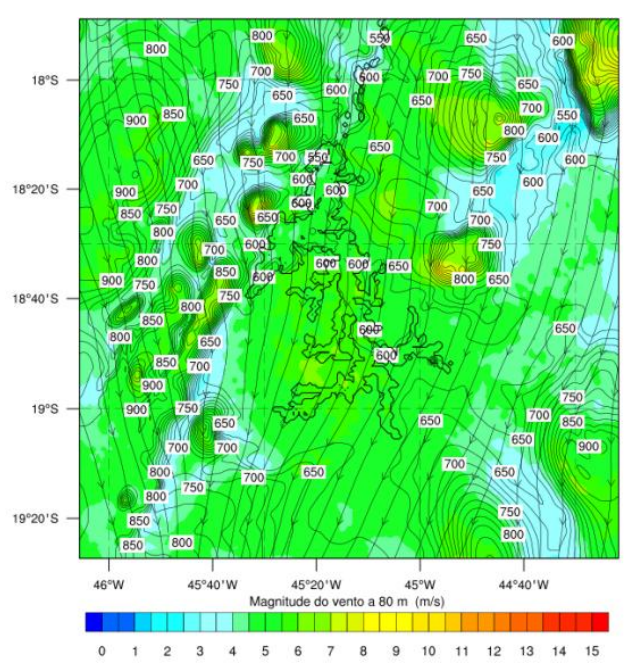

Figura 10- Distribuição de velocidades, dia2 , 80 m, Três Marias, Abril.

\section{Conclusões}

Foram realizadas simulações numéricas utilizando o Modelo WRF V3.5 para os reservatórios de Itumbiara e Três Marias. Foram simuladas uma semana do mês de abril e uma do mês de agosto e obtidas distribuições horizontais de velocidade e potencia eólica bruta nas alturas de 10, 30, 50, 80100 e $120 \mathrm{~m}$ acima do solo. Foram analisadas médias diárias bem como a média semanal do período considerado.

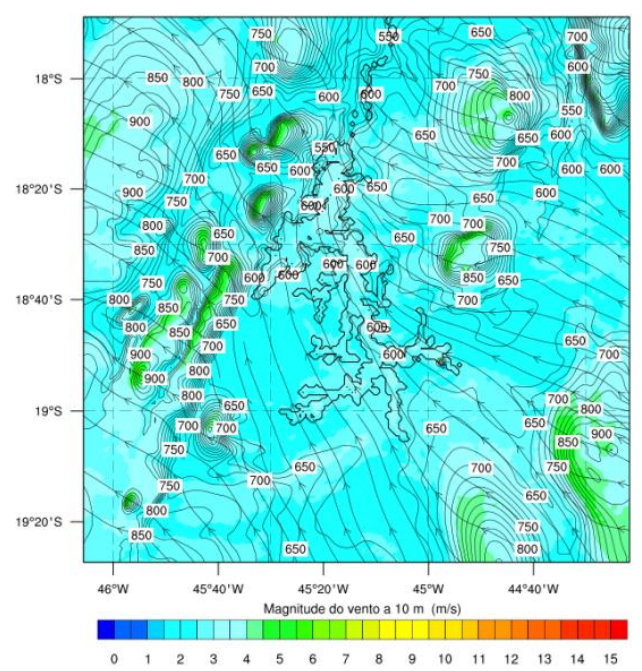

Figura 11 - Distribuição de velocidades, dia 6,10 m, Três Marias, Abril.

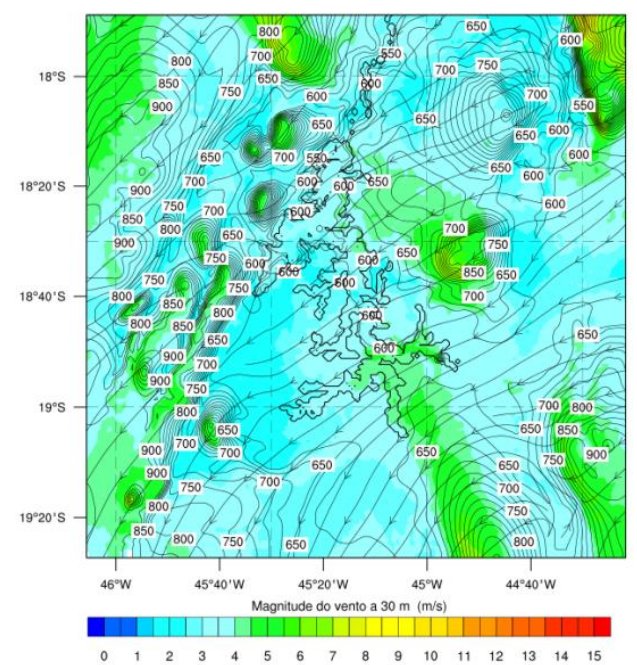

Figura 12 - Distribuição de velocidades, dia 3, 30 m, Três Marias, Agosto.

Alguns testes foram feitos variando o período de acomodação do Modelo entre 6 e 72 horas, e verificou-se uma considerável influência sobre os resultados. Infelizmente, não foi possível definir qual das escolhas era melhor na comparação com dados observados e, por isso optou-se por apresentar apenas os resultados computacionalmente mais baratos, com tempo de 6 horas.

Para Itumbiara, verificou-se que, independente do período e da direção do vento, ocorre a intensificação deste sobre o reservatório. $\mathrm{O}$ efeito é mais marcante próximo ao solo. Evidentemente, em períodos marcados por ventos intensos e alinhados com o reservatório, o efeito de intensificação é mais claro. Em geral, a aceleração passa a ser mais clara em velocidades superiores a 3,0 $\mathrm{m} / \mathrm{s}$ a $10 \mathrm{~m}$, coincidente com a velocidade de entrada (cut-in) da maioria das turbinas moder- 
nas. Em todo o período estudado notou-se que a intensificação dos ventos é devida à influência do conjunto reservatório-relevo. A ação de ambos é essencial para que o efeito ocorra.

Acerca do reservatório de Três Marias concluiu-se que a intensificação dos ventos também esta associada ao conjunto reservatório-relevo. Por exemplo, o efeito de aceleração aparece claramente para ventos $\mathrm{N}$ (alinhados com o eixo principal da represa), mas é muito menor para ventos S. Quando não há canalização pelo relevo, o lago não apresenta tanto efeito intensificador. Assim como em Itumbiara, a aceleração do vento se pronuncia em velocidades superiores a 3,0 m/s

Devido à extensa dimensão do reservatório, as médias semanais mostram regiões de aceleração e outras absolutamente neutras. Não se verificou, porém regiões de desaceleração, nem nos braços perpendiculares ao vento predominante.

Por fim, cabe observar que o relevo, por si só também exerce ação aceleradora do vento, conforme amplamente reconhecido na literatura (Kaimal e Finnigan, 1994, p. ex.) e de magnitude por vezes superior ao efeito do reservatório.

\section{Agradecimentos:}

Agradecimentos a CNPq, CAPES e LGSA.

\section{Referências}

Assireu, A., Pimenta, F., Souza, V. (2011). Assessment of wind power potential of hydroelectric reservoirs. Em: Energy resources: development, distribution and exploitation, pp. 1-28.

Assireu, A., Pellegrini, C.C., Pimenta, F. (2013). Intensificação do vento devido a influências do relevo: evidências a partir de modelos numéricos e medidas in situ. Em: X Workshop Brasileiro. Micrometeorologia. UFSM, Santa Maria, RS.

Bullard, J. E.; Wiggs, G.F.S.; Nahs, D. J. (2000). Experimental study of wind directional variability in the vicinity of a model valley. Em: Geomorphology, 35, 127-143.

INMET, Normas Climatológicas do Brasil (19611990). Ins. Nac. Meteorologia. Disponível em: http://www.inmet.gov.br/portal/index.php?r=cli ma/normaisClimatologicas. Acesso: 29 de abril 1 nnin
Kaimal, J. C. and Finnigan, J. J. (1994). Atmospheric Boundary Layer Flows: Their Structure And Measurement. Oxford Univ. Press, New York, 289 pp.

Lange, B., Larsen, S., Hojstrup, J., Barthelmie, R. (2004). Importance of thermal effects and sea surface roughness for offshore wind resource assessment. Em: Journal of Wind Engineering, 92, 959-988.

Manwell, J. F.; McGowan J. G. and Rogers, A. L. Wind energy explained, John Willey and Sons, West Sussex, England, 590 pp., 2002.

Peña, A., Gryning, S.E. (2008). Charnock's roughness length model and non-dimensional wind profiles over the sea. Em: Bound.-Layer Meteor., 128(2), 191-203.

Peña, A., Gryning, S.E., Hasager, C.B. (2009). Measurements and modeling of the wind speed profile in the Marine Atmospheric Boundary Layer. Em: Bound.-Layer Meteor., 129(3), 479495.

Sathe, A., Gryning, S.E., Peña, A. (2011). Comparison of the atmospheric stability and wind profiles at two wind farm sites over a long marine fetch in the North Sea. Em: Wind Energy, 14(6), 767-780. 